\title{
Results of cytokine research of pregnant women with the risk of premature birth
}

\author{
Ruzieva Nodira KHAKIMOVNA \\ Tashkent Pediatric Medical Institute, Tashkent, Uzbekistan \\ Corresponding author's Email: n-ruzieva@mail.ru
}

\section{ABSTRACT}

Aim. The aim of the research is to study the content of pro-inflammatory and antiinflammatory cytokines of pregnant women with the risk of preterm birth (PB). Methods. Examined 42 women in the third trimester of gestation with the risk of preterm birth. Determination of the cytokine status of IL-1 $\beta$, IL-4, IL-6, IL-8, IL-10 and TNF- $\alpha$ in the serum of peripheral blood was performed by Enzyme immunoassay. Results. The systemic cytokine status was studied in pregnant women with the risk of PB. An imbalance of cytokines has been established, characterized by an increase in the content of pro-inflammatory cytokines and a decrease in anti-inflammatory interleukins, indicating an increased inflammatory response of the organism in the genesis of premature birth. Conclusion. The study of cytokine balance is important to assess the direction of the immune response, as well as the outcome of pregnancy for the mother and fetus. Excessive stimulation of the systemic humoral immune response as a result of increased activity of peripheral pro-inflammatory cytokines and low secretion of anti-inflammatory cytokines are one of the fundamental mechanisms underlying the development of premature birth.

\author{
Original Article \\ PII: S225199391900020-9 \\ Rec. 11 June 2019 \\ Rev. 20 September 2019 \\ Pub. 25 September 2019
}

\section{Keywords}

Preterm birth,

Pro-inflammatory,

Anti-inflammatory

cytokines,

Cytokine status

\section{INTRODUCTION}

The physiological course of pregnancy is accompanied by a certain restructuring of the immune system, which ensures the tolerance of the mother's body to the antigens of the ovum and gestation. It has now become apparent that the protection of the fetus from damaging maternal immune response is based on a complex mechanism and that communication between different steps in the cascade of events is carried out by means of cytokines [1].

In the last decade, active research on the role of cytokines in the development of preterm birth (PB) has been conducted. Being biologically active factors, cytokines, first of all, regulate the development of local defense reactions in tissues with the participation of various types of blood cells, endothelium, connective tissue and epithelium. Cytokines are responsible for all successive stages of development of an adequate response to the introduction of the pathogen, ensuring its localization and removal, and then restoring the damaged tissue structure, no matter where the inflammatory reaction develops [2].

The main role is assigned to the cytokine network, the functioning of which determines the direction of the immune response in inflammation. The importance of cytokines for the life of the body cannot be overestimated. The most studied is their participation in the regulation of immunogenesis, where they are necessary at all stages of the immune response. Cytokines determine differentiation

T-helpers in Th-1 and Th 2-types, which differ in the profile of the cytokines synthesized by them in response to various inductors [3]. Th-1 proinflammatory cytokines produce interleukins: IL-1, IL-3, IL-8; interferons (IFN $\beta$ and $\gamma$ ), tumor necrosis factor (TNF $\alpha$ ), which play an important role in regulating the inflammatory response in the endometrium, limit trophoblast invasion, disrupting its formation. Th-2 is produced by interleukins: IL-4, IL-5, IL-6, IL-10, colony-stimulating factor, etc. -anti-inflammatory cytokines, and IL-10 is also called "suppressor". It is known that Th-1 determines the development of the immune response by cell type, and Th-2 - by humoral type. The physiologically proceeding pregnancy develops with the participation of the Th-2 type of immune response, while there is a certain balance of interaction between Th-1 and Th-2 [4-6]. 
Until now, the main reasons leading to pronounced shifts in the immune system have not been fully studied. At the same time, the study of the state of the immune system during pathological pregnancy can contribute to the pathogenetic substantiation of rational ways of the ante-and intra-natal protection of the fetus and the prevention of complications during childbirth. The aim of study was to investigate the content of pro inflammatory and anti-inflammatory cytokines in pregnant women at risk of developing PB.

\section{MATERIALS AND METHODS}

There were 42 women examined in the third trimester of gestation with the risk of premature birth: recurrent with burdened obstetric history (abortion, preterm birth), with dysbiosis of the vagina and intestines. The study did not include patients with isthmic cervical insufficiency, uterine malformations and myomas, as well as carriers of TORCH infection. All patients came to the clinic with complaints of lower abdominal pain, constipation, poor health, dysuric disorders, and the presence of abnormal discharge from the genital tract.

In $86 \%$ of women in the vaginal contents using polymerase chain reaction diagnostics revealed the presence of pathogenic microflora and mixed infections. The species composition of the microbiocenosis of the vagina and the cervical canal of female patients was characterized by the predominance of the share of coccobacillary flora and gardnerellas. The diagnosis of bacterial vaginosis was established on the basis of clinical and anamnestic indicators, and verified according to light microscopy of a smear from the posterior vaginal fornix and determining the reaction of vaginal secretions ( $\mathrm{pH}$-metry). To describe the microscopic picture of the vaginal biocenosis, light microscopy of smears stained by Gram was performed (Lyumam-P8 microscope, JIOMO, St. Petersburg). The degree of vaginal dysbiosis was determined microscopically by the criteria proposed by Mavzyutov et al. [7]. Later bacteriological cultures were carried out with a quantitative analysis of the microbiocenosis.

Determination of the cytokine status of IL-1 $\beta$, IL-4, IL-6, IL-8, IL-10 and TNF- $\alpha$ in the serum of peripheral blood was performed by Enzyme immunoassay. The data obtained in 24 women with physiological pregnancy were used as controls.

\section{Statistical analysis}

Statistical data processing included determining the arithmetic mean and arithmetic mean error. Reliability of differences was calculated by Student's t-test.

\section{RESULTS}

It was established that by women of the control group the level of cytokine IL-1 $\beta$ in serum was $2.35 \pm 0.18 \mathrm{pg} / \mathrm{ml}$, IL-4: $5.76 \pm 0.44 \mathrm{pg} / \mathrm{ml}$. The serum content of IL-6 was $2.25 \pm 0.17 \mathrm{pg} / \mathrm{ml}$, IL-8: $6.36 \pm 0.58 \mathrm{pg} / \mathrm{ml}$, IL-10: $23.14 \pm 1.57$ $\mathrm{pg} / \mathrm{ml}$, and TNF- $\alpha$ level was in the range of $1.68 \pm 0.13 \mathrm{pg} / \mathrm{ml}$ (Table 1 ).

Analysis of indicators of pregnant women of the main group revealed a significant increase in serum IL-1 $\beta$ production by 6.7 times $(14.6 \pm 0.87 \mathrm{pg} / \mathrm{ml}, \mathrm{P}<0.05)$. IL-1 is an inducible protein, the synthesis of which is necessary for the acute phase response. The main producers of cells are monocytes, macrophages, endothelium and other cells. An excessively high level of IL-1 indicates the possibility of undesirable immunopathological processes. IL-1 is characterized by the ability to stimulate prostaglandin production. Keeping this cytokine low is one of the factors contributing to pregnancy.

By pregnant women with the risk of premature birth, the level of IL-8 was increased 1.6 times (9.98 \pm 0.63 $\mathrm{pg} / \mathrm{ml}$ ) compared with the same indicator in the control group. A high level of spontaneous production of IL-8 may indicate a significant activation of mononuclear phagocyte-producing pro-inflammatory cytokines, which play an important role in the development of immunopathological processes [8].

The obtained data on the increase in IL-1 $\beta$ and IL- 8 are a reflection of the activity of the inflammatory process. An increase in the concentration of pro-inflammatory cytokines suggests that the inflammatory response in a given cohort of pregnant women has systemic manifestations. At the same time, IL-1 stimulates the release of stab leukocytes from the bone marrow, increases the formation and release of collagenase, causes the expression of endothelial-leukocyte adhesive molecules on the surface of endothelial cells and leukocytes, contributes to marginal standing of leukocytes and stimulates the process of their emigration.

As its shown by the results of our studies, pregnant women at risk of premature birth have an increase in serum IL-6 content 2.1 times $(4.83 \pm 0.39 \mathrm{pg} / \mathrm{ml})$ compared with the data of healthy pregnant women $(P<0.05)$. 
Due to a violation of the placental barrier, a large amount of antigenic material of fetal origin enters the mother's circulation. This leads to the induction of an inflammatory response from the maternal immune system with the production of a large amount of IL-6 and TNF-alpha, which causes a high level of apoptosis of the trophoblast. In addition, IL-6 stimulates prostaglandin production, which leads to cervical remodeling and the development of labor activity. IL-6 is used as a marker for predicting preterm labor activity [9].

Table 1. Results of two comparison groups

\begin{tabular}{lcc}
\hline Indicator & $\begin{array}{c}\text { Pregnant women with } \\
\text { the physiological course of gestation, } \\
\mathbf{n = 2 4}\end{array}$ & $\begin{array}{c}\text { Pregnant women with } \\
\text { the risk of premature birth, } \\
\mathbf{n}=\mathbf{4 2}\end{array}$ \\
\hline IL-1 $\mathrm{B}, \mathrm{pg} / \mathrm{ml}$ & $2.35 \pm 0.18$ & $14.6 \pm 0.87^{*}$ \\
IL-2, pg/ml & $11.14 \pm 0.91$ & $7.54 \pm 0.64^{*}$ \\
IL-4, pg/ml & $5.76 \pm 0.44$ & $3.15 \pm 0.23^{*}$ \\
IL-6, pg/ml & $2.25 \pm 0.17$ & $4.83 \pm 0.39^{*}$ \\
IL-8, pg/ml & $6.36 \pm 0.58$ & $9.98 \pm 0.63^{*}$ \\
IL-1O, pg/ml & $23.14 \pm 1.57$ & $7.36 \pm 0.62^{*}$ \\
TNF-a, pg/ml & $1.68 \pm 0.13$ & $3.12 \pm 0.28^{*}$ \\
IgA, g/l & $1.27 \pm 0.13$ & $1.18 \pm 0.12$ \\
IgG, g/l & $12.74 \pm 0.87$ & $16.7 \pm 1.43^{*}$ \\
IgM, g/l & -- & $3.08 \pm 0.29$ \\
\hline In comparison with the control group $(\mathrm{P}<0.05)$. & & \\
\hline
\end{tabular}

According to our data, by pregnant women with the risk of premature birth, the serum level of TNF- $\alpha$ increases 1.9 times $(3.12 \pm 0.28 \mathrm{pg} / \mathrm{ml})$ compared with the control data $(\mathrm{P}<0.05)$. TNF- $\alpha$ is formed by tissue macrophages, monocytes and lymphocytes in the zone of acute inflammation, strengthens the main functions of leukocytes, stimulates the release of histamine by basophils and mast cells, causes activation of fibroblasts, smooth myocytes and vascular endothelium in the inflammation, and induces synthesis of proteins of the acute phase of inflammation. TNF- $\alpha$ hypersecretion leads to a significant increase in the number of apoptotic trophoblast cells, which can be one of the factors contributing to miscarriage [10].

The presence of a strong positive correlation between increased levels of TNF-alpha, IL-1, IL-6, IL-8 and the clinical condition of the examined pregnant women indicates significant impairments in which proinflammatory cytokines enter the systemic circulation, which contributes to pathogenesis of preterm birth. As can be seen from the presented research results, an increase in TNF- alpha and cytokines can serve as markers of inflammation of the vascular endothelium of the uterus, and also indicate a high permeability of the membranes of the fetal membranes, which, in our opinion, is one of the causes of the mechanisms of preterm and amniotic fluid.

Among the risk factors that are considered to cause preterm birth, one of the main ones is infectious. Increased levels of pro-inflammatory cytokines under the influence of infection in the second and third trimesters of pregnancy lead to an increase in the synthesis of prostaglandin by amniotic membranes, contributing to the premature development of labor [11]. It was established that in the normal course of pregnancy, the cytokine status shifts towards immunosuppressive cytokines (IL-2, IL-4, IL-10, TGF- $\beta$ ), which inhibit cellular immunity reactions and stimulate the production of blocking antibodies [12]. In our study, the anti-inflammatory cytokines were: IL-2: $7.54 \pm 0.64 \mathrm{pg} / \mathrm{ml}$, IL-4: $3.15 \pm 0.23 \mathrm{pg} / \mathrm{ml}$, IL-10: $7.36 \pm 0.62 \mathrm{pg} / \mathrm{ml}$ that is, respectively, significantly 1.5 times, 1.8 and 3.1 times lower than the corresponding indicators of the control group. The most informative is the level of IL-10, insufficient production of this anti-inflammatory cytokine can happen to be a marker of the risk of developing preterm birth.

\section{CONCLUSION}

Our research results suggest that the study of cytokine balance is important to assess the direction of the immune response, as well as the outcome of pregnancy for the mother and fetus. Excessive stimulation of the systemic humoral immune response as a result of increased activity of peripheral pro-inflammatory cytokines and low secretion of anti-inflammatory cytokines are one of the fundamental mechanisms underlying the development of preterm birth. 


\section{Acknowledgements}

This work was supported by Department of Obstetrics and Gynecology, Pediatric Gynecology, Uzbekistan.

\section{Authors' contributions}

All authors contributed equally to this work.

\section{Competing interests}

The authors declare that they have no competing interests.

\section{REFERENCES}

1. Musakhodzhaeva DA. Immunobiological indicators in women outside and during pregnancy are normal and when exposed to adverse factors. The Doctoral Program in Biological Sciences. Tashkent. 2010. pp. 34

2. Stashkevich DS, Yu FY, Burmistrova AL. Actual issues of immunology: cytokine system, biological significance, genetic polymorphism, determination methods [Aktual'nyye voprosy immunologii: sistema tsitokinov, biologicheskoye znacheniye, geneticheskiy polimorfizm, metody opredeleniya]. Google Scholar

3. Kashtalyan OA. Features of production of cytokines and immunoglobulins in pregnant women. J. Medical. Sci. 2009. 4: 79-82. [Russion]

4. Alieva DA, Mamutova GA, Musakhodzhaeva DA. The dynamics of immunological parameters in case of miscarriage of pregnancy of infectious genesis before and after treatment. Nazariy Varya Clinics Tibbiet Journal. 2006; 3: 46-49. [Russion]

5. Zufarova ShA, Yuldasheva DA, Mirzaeva NB et al. Indicators of the cytokine status of pregnant women with chronic pyelonephritis. Dermatology and Reproductive News. Health. 2002; 2: 45-46. [Russion]

6. Fayzyrakhmanova MM, Khairutdinova NKh, Nazarova KYa. Some indicators of cytokine status in pregnant women: scientific publication. News of Dermatovenereology and Reproductive Health. 2008. № 3: 102-103. [Russion]

7. Mavzutov AR, Tsvetkova AV, Nuretdinova L.A. Clinical and laboratorz diagnostics; Russian Federation. Moscow. 2015; 60(6): 41-45. [Russion]

8. Kashtalyan OA, Pristrom MS. Cytokine profile evaluation in pregnant women. J. Cytocines and Inflammation. Russian Federation. 2019; 5(65): 35-37.

9. Ushakova GA, Petrich LN. Modern ideas about the mechanisms of development of labor. Review. Mother and child in Kuzbass. Kemerovo State Medical Academy, Kemerovo. 2016; 2: 65.

10. Pitirimova LN. Immunological and genetic predictors of recurrent miscarriage topic Author's abstract. Dissertation of doctor of medicine. DSC Journal. St. Petersburg, 2014.

11. Moroz VV, Perepelitsa SA, Golubev AM, Golubev MA. Cytokines are markers of immunoreactivity in preterm infants. General resuscitation, 2011, five. https://doi.org/10.15360/1813-9779-2011-5-36

12. Nefedova DD, Linde VA, Levkovich MA. Immunological aspects of pregnancy. Meditsinskiy vestnik Yuga Rossii. 2013; 4:16-21. Google Scholar 